\title{
Multiscale Characterization of bcc Crystals Deformed to Large Extents of Strain
}

J.N. Florando,M.M LeBlanc, D.H. Lassila, V.V. Bulatov, M. Rhee, A. Aresenlis, R. Becker, J.W. Morris JR., and K.R. Magid.

Feburary 20, 2007

LDRD Final Report 


\section{Disclaimer}

This document was prepared as an account of work sponsored by an agency of the United States Government. Neither the United States Government nor the University of California nor any of their employees, makes any warranty, express or implied, or assumes any legal liability or responsibility for the accuracy, completeness, or usefulness of any information, apparatus, product, or process disclosed, or represents that its use would not infringe privately owned rights. Reference herein to any specific commercial product, process, or service by trade name, trademark, manufacturer, or otherwise, does not necessarily constitute or imply its endorsement, recommendation, or favoring by the United States Government or the University of California. The views and opinions of authors expressed herein do not necessarily state or reflect those of the United States Government or the University of California, and shall not be used for advertising or product endorsement purposes.

\section{Auspices Statement}

This work was performed under the auspices of the $U$. S. Department of Energy (DOE) by the University of California, Lawrence Livermore National Laboratory (LLNL) under Contract No. W-7405-Eng-48. The project (04-ERD-036) was funded by the Laboratory Directed Research and Development Program at LLNL. 


\title{
FY06 LDRD Final Report
}

\section{Multiscale Characterization of bcc Crystals Deformed to Large Extents of Strain}

\author{
LDRD Project Tracking Code: 04-ERD-036
}

\section{Jeff Florando, Principal Investigator}

\author{
Co-investigators: Mary Leblanc, David Lassila, Vasily Bulatov, Moono \\ Rhee, Athanasios (Tom) Arsenlis, Richard Becker, J. William Morris \\ Jr. (UCB), and Karen Magid (UCB)
}

\begin{abstract}
In an effort to help advance the predictive capability of LLNL's multiscale modeling program a new experimental technique has been developed to provide high fidelity data on metallic single crystals out to relatively large extents of strain. The technique uses a '6 Degrees of Freedom' testing apparatus in conjunction with a 3-D image correlation system. Utilizing this technique, a series of experiments have been performed that reveal unexpected behavior which cannot be explained using traditional crystal plasticity theory. In addition, analysis and characterization techniques have also been developed to help quantify the unexpected behavior. Interactions with multiscale modelers include the development of a possible mechanism that might explain the anomalous behavior, as well as the discovery of a new 4-node dislocation junction.
\end{abstract}

\section{Introduction/Background}

Understanding and simulating the plastic, or non-reversible, deformation of bodycentered cubic (bcc) metals is a major component of DNT's Stockpile Stewardship Program and is intended to simulate future NIF experiments. The DNT computer code simulations however, require additional experimental data in order to develop and validate the multiscale crystal plasticity models currently used. Previous studies [1] have focused on relatively small extents for strain $(<1 \%)$, where model development must begin. Recent advances in model development and simulation capability [2,3] have lead to a need for larger strain data. Therefore, the objectives of this project are to provide large strain data to further the understanding of the underlying mechanism in bcc metals, which will lead to the validation of dislocation dynamics (DD) simulations and the development of crystal plasticity models.

\section{Research Path}

Using previous studies [1] and a review of the literature as a guide [4-8], it was determined that the first step was to develop a high fidelity experimental technique utilizing a 3-D image correlation system to measure the strain or deformation in the 
material. The advantage to using the image correlation system is the wealth of additional information available over previous strain measurements. In addition, new characterization and analysis techniques were developed to extract the necessary information to aid in understanding the underlying behavior.

The initial experiments revealed surprising results which could not be explained with the current theory. Utilizing the experimental and analysis techniques developed under this LDRD, a series of experiments were performed to help pinpoint the underlying deformation mechanisms. In addition, interaction with the theoretical modelers has lead to some interesting discoveries and some possible mechanisms to explain the behavior.

\section{Results/Technical Outcome}

\section{Experimental technique}

As outlined in the research path, the first step in the process is to establish a high fidelity experimental technique to measure the deformation response to relatively large strains. The experimental technique developed utilized a '6 Degree of Freedom' (6DOF) testing apparatus in combination with a commercial 3-D digital image correlation system. The image correlation system has two distinct advantages over traditional techniques in its ability to measure full-field strains and displacements out to large extents of strain (20\%-30\%). A paper (UCRL-JRNL-213561) written under this LDRD and published in Experimental Techniques [9], details the comparison of image correlation to the traditional and accurate, strain gage, as well as discuses the application to single crystal experiments.

The advantage of the ' 6 Degree of Freedom' testing apparatus [1], is the unconstrained deformation of the sample, which is accomplished by loading the sample through a half sphere, and attaching the sample to a translation platen that sits on ball bearings. A paper (UCRL-JRNL-221336) accepted for publication in Metallurgical and Materials Transactions [10],details and validates the 6DOF testing apparatus, its application to single crystal experiments, and discuses some surprising experimental results on zinc (hexagonal close-packed (hcp) crystal structure).

The Metallurgical and Materials Transactions paper [10] also includes a finite element analysis (FEA) of the stress state during a 6DOF test. Utilizing this information it was determined that in order to maintain a nominally uniaxial stress state, the samples needed to be remachined after a few percent strain. A method to remachine and maintain the orientation of the samples was developed. The results, figure 1a, show the stress-strain behavior of a molybdenum (bcc crystal structure) sample that was tested to $2.5 \%$ strain, remachined, and then tested an additional $2.5 \%$ for a total of 5\% strain. The results also show that the overall behavior of the sample is maintained after the remachining processes. Also, figure $1 \mathrm{~b}$ shows that displacement of the bottom of the sample during the test. Based on the orientation of this sample, traditional Schmid theory [11] would predict that the bottom of the sample would move in the +x-direction, but results show that the sample moves in the $-\mathrm{x}$, $+\mathrm{y}$-direction. Figure 1 also shows that this anomalous behavior continues after the remachining process. 


\section{Analysis of data}

Once the experimental technique has been established and verified, the next step is to develop techniques to analyze the data. It should be noted that the 3-D image correlation system allows access to a wealth of information on the deformation of the sample that was not available to previous researchers in the field. The previously mentioned Experimental Techniques and Metallurgical and Materials Transactions papers have some discussion on how the image correlation data is used to extract pertinent information on the deformation response of single crystals. In order to connect with the relevant parameters and results used in the simulations, additional analysis techniques were needed. One of the main parameters needed is the slip system activity for a given deformed single crystal. The Philosophical Magazine Letter paper (UCRL-JRNL219533) contains an analysis which utilizes the wealth of information from the image correlation technique, to calculate the slip system activity in deformed zinc crystals [12]. The analysis shows activity on unexpected slip systems. This analysis has also been applied to deformed molybdenum, figure 2, and also reveals activity on unexpected slip systems.

\section{Experiments and Characterization}

Since the analysis and initial experiments (see previously mentioned papers) show unexpected behavior, additional experiments and characterization of the deformed samples is necessary. While the goal of this project is to understand the behavior of bcc crystals, experiments on molybdenum (bcc), zinc (hcp), and copper (face-centered cubic (fcc crystal structure)), all reveal unexpected behavior which might be universal to all three main classes of metals.

Therefore, it was determined that a series of experiments would be performed on $\mathrm{Cu}$ due to the more straightforward crystal structure (it is truly closed-packed) and the large body of data that already exists on the deformation of Cu single crystals. A summary of the experiments on $\mathrm{Cu}$, as well as a description of the unexpected behavior is contained in a paper that has been submitted to Scripta Materialia (UCRL-JRNL-228160).

In addition to the experimental series, characterization of the deformed material is needed. For this aspect of the work, we collaborated with Prof. J. William Morris Jr. at UC Berkeley and his graduate student Karen Magid. Karen’s work involved characterizing the deformed Mo and Cu material using the x-ray microdiffraction beamline at the Advanced Light Source (ALS) at Lawrence Berkeley National Laboratory. Karen work revealed some interesting structure in deformed Mo and $\mathrm{Cu}$, and a summary of the work is contained in an MRS proceeding paper (UCRL-PROC-208322) [13], and a paper to be submitted to Philosophical Magazine (UCRL-JRNL-228370).

Also, the Cu deformed microstructure has been analyzed by Karen and Luke Hsiung, using the Transmission Electron Microscope (TEM) to compare with the ALS data. A TEM picture of the dislocation structure is shown in figure 3. These pictures show activity on multiple slip planes which is consistent with our experimental results, and contradictory with what the theory would predict.

\section{Connection with modelers:}

The connection between the experiments and the simulations is essential to understanding the anomalous behavior seen in the experiments. A theory which might 
explain this behavior is based on dislocation interactions. An atomistic simulation by Vasily Bulatov and Wei Cai [14], showed that in bcc materials, dislocations can interact and form a network of [001] junctions as shown in Fig. 4a. The mobility of these junctions is higher than the mobility of the individual dislocations. This enhanced mobility cannot be predicted from traditional theories, which are based solely on the movement of individual dislocations. Also, Moon Rhee performed a DD simulation which showed, Fig. 4b, that for the plane observed to have the most activity in the Mo experiments, the physical geometry of the dislocations was consistent with the formation of the [001] junction. This and other potential models to explain the unexpected behavior are currently being developed for incorporation into the DD code.

In a coordinated effort between the DD simulation development team and this LDRD project the underlying physics of high temperature deformation of bcc metals was investigated. Large strain experiments on symmetrically oriented samples, [001] and [110], at 500K were performed. Both the experiment and the DD results, Fig.5, show a large difference in the strain hardening rate between the [001] and [110] samples. This orientation dependence can be explained with 4-node junctions, which is an effect first seen in the DD code and verified experimentally. A detailed description of these 4-node junctions and their implications to strain hardening has been published in Nature (UCRLJRNL-222317) [15]. In addition, qualitatively, the shape of the stress-strain curves between the experiments and the simulations are very similar, which suggests that the underlying physics are correct. The discrepancy in the stress levels can probably be explained by the mobility functions used in the simulations.

\section{Exit Plan}

The accomplishments of this LDRD project will advance the predictive capabilities of the DD simulations and crystal plasticity models. However, additional work will likely be needed to reach the ultimate goal of the multiscale modeling program. One area which will become more important as DD simulations begin to achieve even larger strains is the understanding the local lattice rotations. X-ray microdiffraction experiments at ALS have the necessary resolution to map the evolution of the rotations as a function of deformation and have revealed local rotations in our deformed sample. In addition, experimental data that links the underlying microstructure to the macroscopic properties is critical input into the mechanistic based crystal plasticity models.

Since the accomplishment and goals of this proposal are well aligned with DNT's "Dynamics of Metal Program" funding from DNT has been allocated to continue some of this work.

\section{Conclusions}

Under this LDRD, a testing technique has been developed, utilizing a 6DOF testing apparatus and a 3-D image correlation system to provide previously unattainable data on single crystal deformation out to relatively large strains. This work has been described in the Experimental Techniques and Metallurgical and Materials Transactions papers. Using this technique as series of experiments has been preformed which show that the deformation behavior of single crystals cannot be completely explained using traditional theories, as described in a paper submitted to Scripta Materialia. Additional analysis and characterization techniques have been developed and utilized to help explain the 
unexpected behavior. The analysis is contained in the Philosophical Magazine Letters Paper, and the x-ray microdiffraction work is described in an MRS proceedings paper and an article to be submitted to Philosophical Magazine. Finally interactions with the multiscale modelers include the discovery of a 4-node dislocation junction, which was the topic of an article published in Nature, as well as some possible mechanisms to describe the unexpected behavior.

A summary of the publications under this LDRD are as follows:

- $\quad$ K.R. Magid, E.T. Lilleodden, N. Tamura, J.N. Florando, D.H. Lassila, M.M.Leblanc, R.I. Barabash, J. W. Morris Jr., "X-ray microdiffraction characterization of deformation heterogeneities in BCC crystals” Neutron and XRay Scattering as Probes of Multiscale Phenomena. Symposium. Materials Research Society. pp. 181-6. Warrendale, PA, USA. (2004). UCRL-PROC208322

- V. V. Bulatov*, M. C. Bartelt*, L. L. Hsiung*, M. Tang*, A. Arsenlis*, W. Cai*†, J.N. Florando*, M. Hiratani*, M. Rhee*, G. Hommes*, T. Pierce*, T. Rubia* “Dislocation multi-junctions”, Nature, 440, 1174 (2006). UCRL-JRNL222317

- M.M. LeBlanc, J.N. Florando, D.H. Lassila, T. Schmidt, and J. Tyson, “Image Correlation Applied to Single Crystal Plasticity Experiments and Comparison to Strain Gage Data” Experimental Techniques, 30, 33 (2006). UCRL-JRNL213561

- J.N. Florando, M.Rhee, A. Arsenlis, M.M. LeBlanc, and D.H. Lassila, "Calculation of the Slip System Activity in Deformed Zinc Single Crystals Using Digital 3-D Image Correlation Data”, accepted by Philosophical Magazine Letters, 86, 795 (2006). UCRL-JRNL-219533

- D. H. Lassila, M.M. LeBlanc, and J.N. Florando "Single Crystal Deformation Experiments using a “6 Degrees of Freedom” Apparatus”, Metallurgical and Materials Transactions, in press, accepted for publication Jan. 2007. UCRLJRNL-221336

- J.N. Florando, M.M. LeBlanc, and D.H. Lassila, “'Multiple slip in copper single crystals deformed in compression under uniaxial stress'” submitted to Scripta Materialia, (Dec. 2006). UCRL-JRNL-228160

- $\quad$ K.R. Magid, J.N. Florando, D.H. Lassila, M.M. LeBlanc, N. Tamura and J.W. Morris, Jr., "Mesoscale Heterogeneity in the Plastic Deformation of a Copper Single Crystal”. submitted to Philosophical Magazine. UCRL-JRNL-228370 


\section{Acknowledgements}

The success of an LDRD depends on the work of many people, and the authors would like to especially thank Ann Bliss for Laue characterization of the crystals, and Barry Olsen for purification of the crystals. In addition the authors would like to thank Dr. John Hirth for his enlightening discussions and suggestions regarding this work. Finally, we would like to thank Dr. Elaine Chandler and Dr. Kim Budil, who headed up DNT's Dyanmics of Metals Program during the duration of this LDRD, for their support and guidance. This work was performed under the auspices of the U. S. Department of Energy (DOE) by the University of California, Lawrence Livermore National Laboratory (LLNL) under Contract No. W-7405-Eng-48. The project (04-ERD-036) was funded by the Laboratory Directed Research and Development Program at LLNL.

\section{References}

[1] D.H. Lassila, M.M. LeBlanc, G.J. Kay, J. Eng. Mat. \& Tech.-Trans. of the ASME 124 (2002) 290.

[2] R. Madec, B. Devincre, L.P. Kubin, Physical Review Letters 89 (2002) 2555081.

[3] P. Hahner, M. Zaiser, Materials Science \& Engineering A (Structural Materials: Properties, Microstructure and Processing) A272 (1999) 443.

[4] J.H. Hoke, R. Maddin, J. Mech. and Phys. of Solids. 5 (1956) 26.

[5] J. Richter, Physica Status Solidi 40 (1970) 565.

[6] J. Richter, Physica Status Solidi 46 (1971) 203.

[7] G.J. Irwin, F. Guiu, P.L. Pratt, Physica Status Solidi A 22 (1974) 685.

[8] J.W. Christian, Metallurgical Transactions A (Physical Metallurgy and Materials Science) 14A (1983) 1237.

[9] M.M. LeBlanc, J.N. Florando, D.H. Lassila, J. Tyson III, T. Schmidt, Exp. Tech. 30 (2006) 33.

[10] D.H. Lassila, M.M. LeBlanc, J.N. Florando, Met. and Mat. Trans. A. (in press)

[11] E. Schmid, I.W. Boas, Plasticity of Crystals, Chapman and Hall LTD, London, 1950.

[12] J.N. Florando, M. Rhee, A. Arsenlis, M.M. LeBlanc, D.H. Lassila, Phil. Mag. Let. 86 (2006) 795.

[13] K.R. Magid, E.T. Lilleodden, N. Tamura, J.N. Florando, D.H. Lassila, M.M. LeBlanc, R.I. Barabash, J.W.J. Morris, Neutron and X-Ray Scattering as Probes of 
Multiscale Phenomena. Symposium. Materials Research Society. 2005, pp. 181-6. Warrendale, PA, USA.,(2004)

[14] V.V. Bulatov, C. Wei, Physical Review Letters 89 (2002) 1155011.

[15] V.V. Bulatov, L.L. Hsiung, T. Meijie, A. Arsenlis, M.C. Bartelt, C. Wei, J.N. Florando, M. Hiratani, M. Rhee, G. Hommes, T.G. Pierce, T.D. de la Rubia, Nature 440 (2006) 1174. 


\section{Figures}

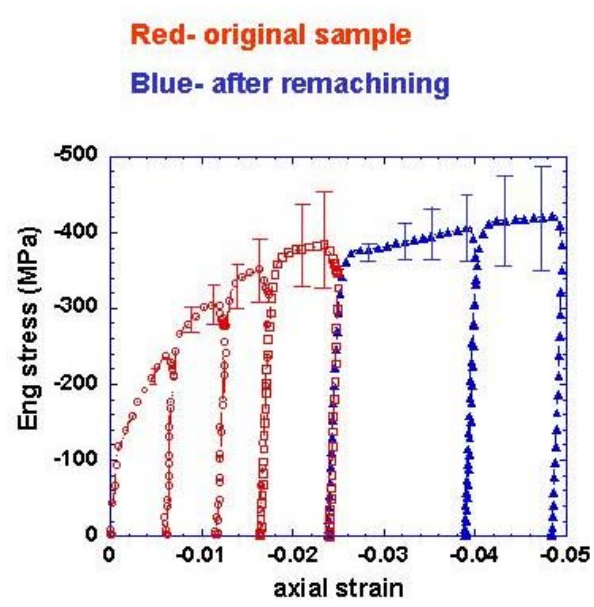

a)

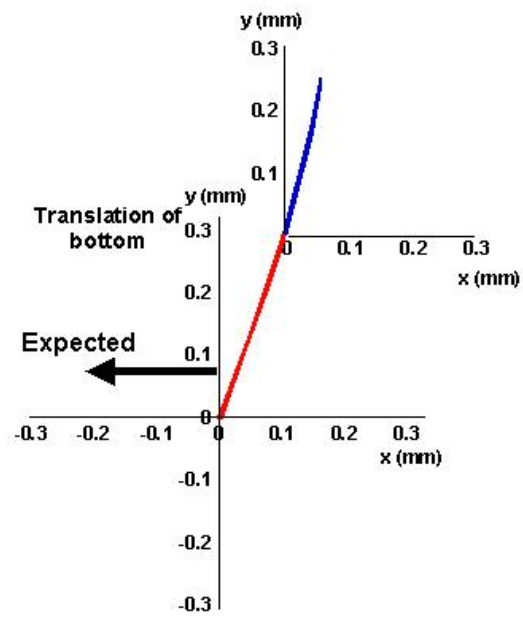

b)

Figure 1- Experimental results on Mo sample before and after remachining. a) Stress-strain curve showing behavior out to $5 \%$ strain. b) Translation of the bottom of the sample, inconsistent with predicted theory.

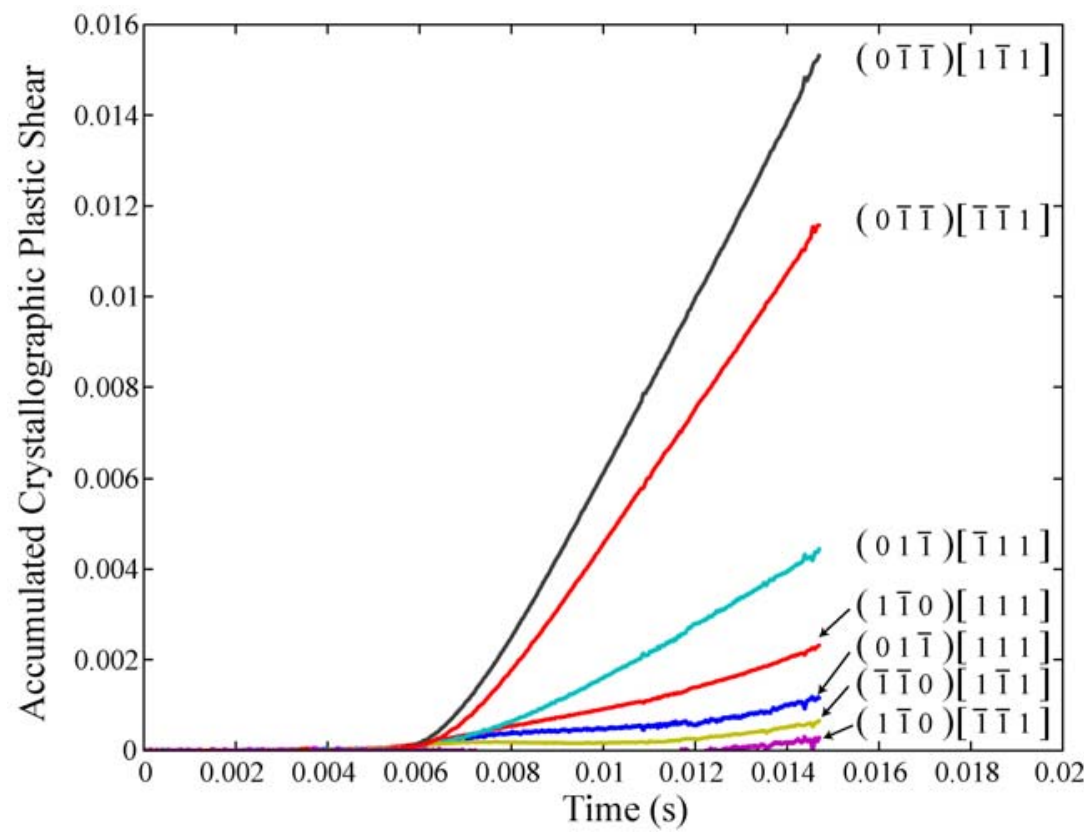

Figure 2- Calculated slip system activity for a deformed Mo sample showing activity on multiple slip systems. The expected primary slip system shows no activity. These activities cannot be explained using traditional theories. 


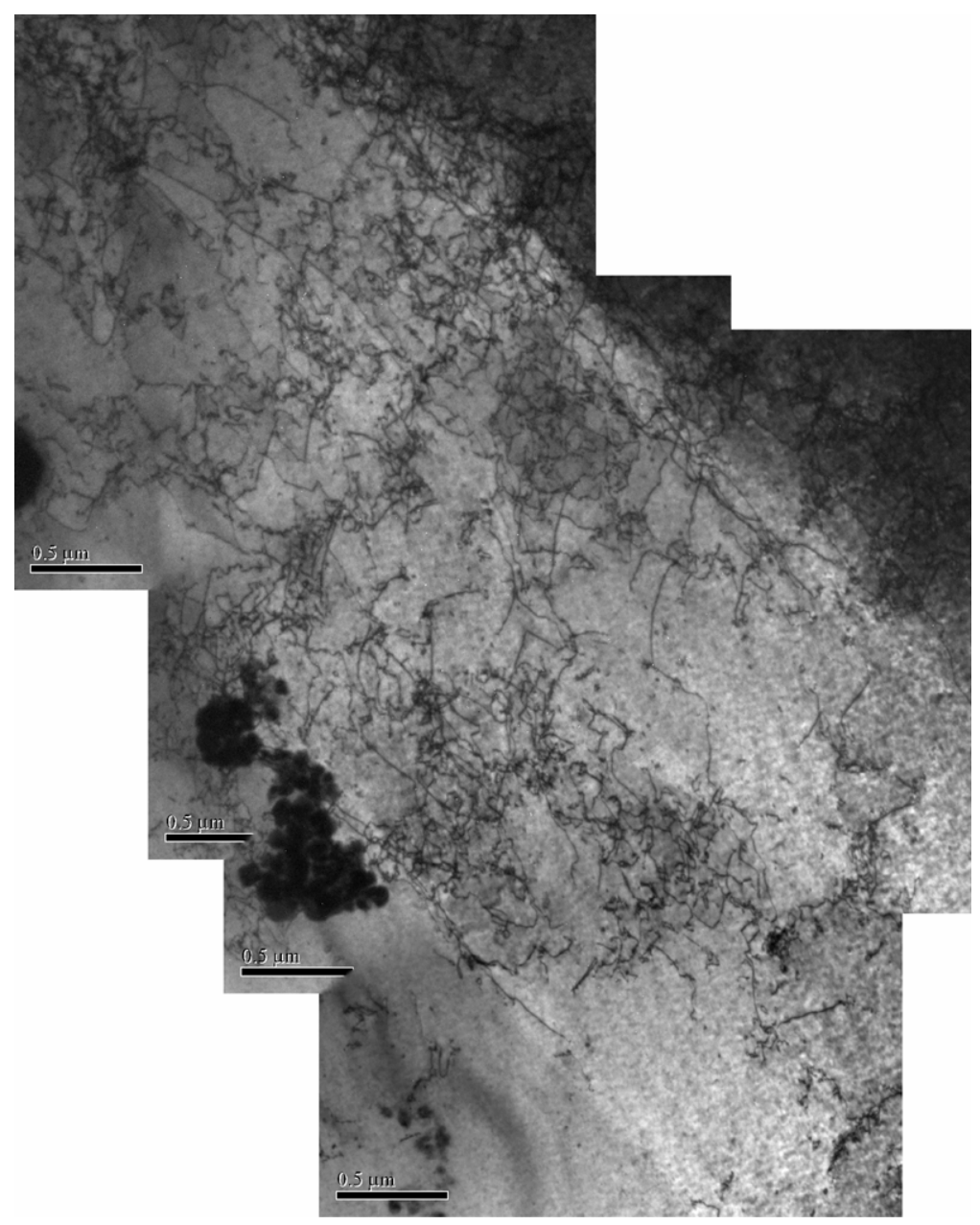

Figure 3- Transmission Electron Microscope (TEM) photo showing the dislocation structure in Cu. These structures are similar and consistent with the overall structures seen in the ALS experiments.

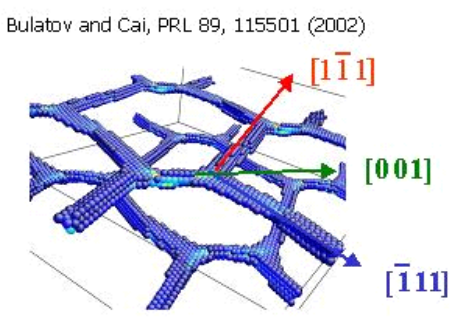

a)

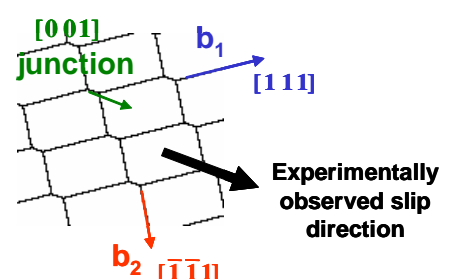

b)

Figure 4- Simulations of the[ 001] junction a) Atomistic simulation showing a network of 001 junctions. The enhanced mobility is due to kink formation and propagation at the junction b) DD simulation showing the formation of the [001] junction in the experimentally observed plane with the highest activity. 


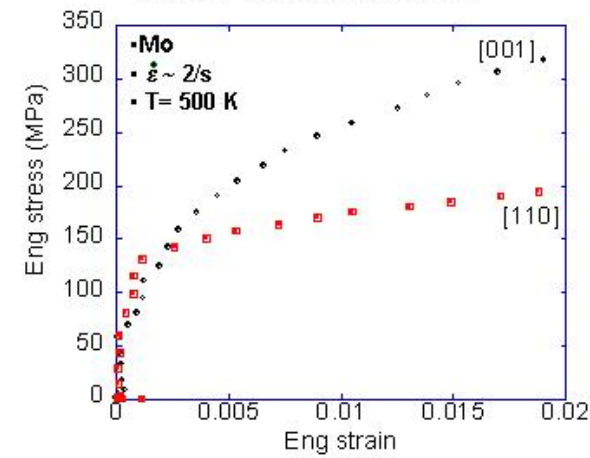

a)

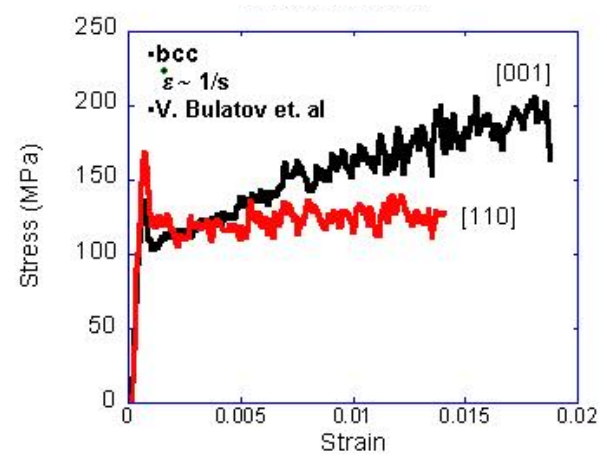

b)

Figure 5- Comparison of experimental results with ParaDis Dislocation Dynamics simulations. a)Experimental result b)DD simulation 